\title{
Correction to: On Milstein approximations with varying coefficients: the case of super-linear diffusion coefficients
}

\author{
Chaman Kumar ${ }^{1} \cdot$ Sotirios Sabanis $^{2}$ (])
}

Published online: 8 October 2019

(c) Springer Nature B.V. 2019

\section{Correction to: BIT Numerical Mathematics https://doi.org/10.1007/s10543-019-00756-5}

In the originally published version of this article, the acknowledgement was unfortunately not yet in the final version. It should read as follows:

Acknowledgements Some progress in this work was made when the first author was working as a Visiting Scientist at the Indian Statistical Institute (ISI), Delhi Center. The major part of the work was done when the first author was visiting the School of Mathematics, University of Edinburgh in June 2018 which was financially supported by Faculty Initiation Grant (FIG) and Professional Development Allowance (PDA) from the Indian Institute of Technology (IIT) Roorkee. The first author also acknowledges the hospitality received by the School of Mathematics, University of Edinburgh during his research visit in June 2018.

We apologize for this error.

Publisher's Note Springer Nature remains neutral with regard to jurisdictional claims in published maps and institutional affiliations.

The original article can be found online at https://doi.org/10.1007/s10543-019-00756-5.

Sotirios Sabanis

S.Sabanis@ed.ac.uk

Chaman Kumar

c.kumarfma@iitr.ac.in

1 Department of Mathematics, IIT Roorkee, Roorkee, India

2 School of Mathematics, University of Edinburgh, Edinburgh, UK 